IFUM 441/FT

February 1993

\title{
SOLITONS IN TWO-DIMENSIONAL TOPOLOGICAL FIELD THEORIES
}

\author{
S. PENATI \\ Dipartimento di Fisica dell'Università di Milano and \\ INFN, Sezione di Milano, Via Celoria 16, I-20133 Milano, Italy \\ M. PERNICI \\ INFN, Sezione di Milano, Via Celoria 16, I-20133 Milano, Italy \\ D. ZANON \\ Dipartimento di Fisica dell'Università di Milano and \\ INFN, Sezione di Milano, Via Celoria 16, I-20133 Milano, Italy
}

\begin{abstract}
We consider a class of $N=2$ supersymmetric non-unitary theories in twodimensional Minkowski spacetime which admit classical solitonic solutions. We show how these models can be twisted into a topological sector whose energy-momentum tensor is a BRST commutator. There is an infinite number of degrees of freedom associated to the zero modes of the solitons. As explicit realizations of such models we discuss the BRST quantization of a system of free fields, while in the interacting case we study $N=2$ complexified twisted Toda theories.
\end{abstract}


In the past few years progress has been made in the study of non-critical strings. In particular the $c<1$ theories are understood by now in terms of rather different techniques, i.e. conformal field theory [1], matrix models [2], topological field theory [3]. In the topological field theory framework one shows that correlation functions of the observables are independent of the two-dimensional metric. Therefore no modes propagate on the world-sheet manifold and the only relevant quantities are the zeromodes of the instantons. A distinguished class of these models is described in euclidean space by $N=2$ supersymmetric lagrangians which can be twisted into a topological sector using the $U(1)$ invariance of the theory. It has been shown that these systems coupled to topological gravity correspond to string theories with $c \leq 1$ [3, 4, 5].

In this letter we study a class of $N=2$ supersymmetric theories in Minkowski spacetime and address the issue of their topological formulation. In Minkowski space a theory can be twisted into a topological, BRST invariant sector, if the Lorentz group can be combined with a $O(1,1)$ symmetry. We show that in order to implement the $O(1,1)$ invariance one has to consider models which have $N=2$ complexified supersymmetries, and this in turn can be realized only in a non-unitary lagrangian description. However the problem of non-unitarity is naturally solved in the topological twisted version of the theory. In fact the existence of a BRST charge provides the standard mechanism which allows to eliminate ghost-like fields from the physical spectrum. The topological symmetry enforces no propagation on the two-dimensional worldsheet and the remnant degrees of freedom are given by the zero modes which determine the quantum numbers of the physical observables. A simple, completely solvable example of such theories is provided by a system of free fields. We discuss the physical spectrum and find that all particles excitations are cohomologically trivial. There is a single massless field propagating in the target spacetime. If the free fields are compactified, the winding solitons are the only physical states.

Then we switch on the interaction and introduce a potential with an infinite number of critical points. The resulting topological theories have infinite degrees of freedom given by the zero modes of the solitons which interpolate between different extrema of the potential. These systems can be explicitly realized by $N=2$ complexified affine Toda theories, which indeed are described by non-unitary lagrangians of the general form that allows the topological Minkowskian twist. It has been shown that affine complex Toda theories admit solitonic solutions [6, 7, 8] which, in spite of the non-unitarity of the action, have real energy and momentum. We consider the corresponding topological sector and show that there survives an infinite number of solitonic configurations that can be studied at the quantum level in a weak coupling approximation. These are the issues that we elucidate in this letter. 
First we set our notations and conventions: $N=1$ superspace is parametrized by two light-cone Minkowski coordinates $z, \bar{z}$

$$
\begin{aligned}
& z \equiv x^{+}=\frac{1}{\sqrt{2}}\left(x^{0}+x^{1}\right) \quad \bar{z} \equiv x^{-}=\frac{1}{\sqrt{2}}\left(x^{0}-x^{1}\right) \\
& \partial \equiv \partial_{z}=\frac{1}{\sqrt{2}}\left(\partial_{0}+\partial_{1}\right) \quad \bar{\partial} \equiv \partial_{\bar{z}}=\frac{1}{\sqrt{2}}\left(\partial_{0}-\partial_{1}\right) \quad \square=2 \partial \bar{\partial}
\end{aligned}
$$

and by two spinor coordinates $\theta, \bar{\theta}$, with $\theta^{*}=-\theta, \bar{\theta}^{*}=\bar{\theta}$. Complex conjugation for the product of spinors is defined as $(\chi \psi)^{*}=\psi^{*} \chi^{*}$. We introduce covariant spinor derivatives

$$
D=\partial_{\theta}+i \theta \partial \quad \bar{D}=\partial_{\bar{\theta}}-i \bar{\theta} \bar{\partial}
$$

which satisfy $\{D, \bar{D}\}=0, D^{2}=i \partial$ and $\bar{D}^{2}=-i \bar{\partial}$. Finally, a generic $N=1$ superfield is denoted by

$$
\Phi=\phi+\frac{1}{\sqrt{2}} \theta \psi+\frac{1}{\sqrt{2}} \bar{\theta} \bar{\psi}+\theta \bar{\theta} F
$$

We start by considering a class of field theories in two-dimensional Minkowski spacetime described in terms of $2 n$ complex $N=1$ superfields, with general action

$$
S=\frac{1}{\beta^{2}} \int d^{2} z d^{2} \theta\left[K_{i j} D \Phi_{i}^{(-)} \bar{D} \Phi_{j}^{(+)}+V\left(\Phi^{(+)}\right)+V\left(\Phi^{(-)}\right)+\text {h.c. }\right]
$$

where $d^{2} z=d z d \bar{z}, d^{2} \theta=\bar{D} D, \beta$ is a coupling constant and $K_{i j}$ is an invertible, symmetric, constant $n \times n$ real matrix. We emphasize that no reality conditions are imposed on the superfields and the theory is not unitary.

In addition to the explicit $N=1$ supersymmetry the action in eq.(14) possesses a second supersymmetry. The supersymmetry transformations on the fields are respectively

$$
\begin{aligned}
\delta \Phi_{i}^{( \pm)} & =\left(\varepsilon_{L} Q_{L}^{+}+\varepsilon_{R} Q_{R}^{+}\right) \Phi_{i}^{( \pm)} \\
\delta \Phi_{i}^{( \pm)} & =\left(\zeta_{L} Q_{L}^{-}+\zeta_{R} Q_{R}^{-}\right) J \Phi_{i}^{( \pm)}
\end{aligned}
$$

where the charges

$$
\begin{array}{ll}
Q_{L}^{+}=i\left(\partial_{\theta}-i \theta \partial\right) & Q_{R}^{+}=i\left(\partial_{\bar{\theta}}+i \bar{\theta} \bar{\partial}\right) \\
Q_{L}^{-}=\partial_{\theta}+i \theta \partial=D & Q_{R}^{-}=\partial_{\bar{\theta}}-i \bar{\theta} \bar{\partial}=\bar{D}
\end{array}
$$

satisfy the supersymmetry algebra $\left(Q_{L}^{ \pm}\right)^{2}=i \partial,\left(Q_{R}^{ \pm}\right)^{2}=-i \bar{\partial}$ and $\left\{Q^{+}, Q^{-}\right\}=0$. The operator $J$ acts on the fields as $J \Phi_{i}^{( \pm)}= \pm \Phi_{i}^{( \pm)}$. Unlike in standard $N=2$ theories, the parameters $\varepsilon, \zeta$ which appear in eq.(5) are complex in general, so that the action in eq.(4) is invariant under a complexified $N=2$ algebra. This implies that the usual $U(1)$ 
invariance is extended to a $U(1) \otimes O(1,1)$. This will play a crucial role in the definition of the twisted theory and in the construction of the BRST charge.

The complex theories under consideration can be formulated in $N=2$ superspace embedding the $N=1$ superfields into chiral and antichiral $N=2$ supermultiplets [9]. One introduces $N=2$ spinor coordinates $\theta_{+}, \theta_{-}, \bar{\theta}_{+}, \bar{\theta}_{-}$and corresponding covariant spinor derivatives satisfying

$$
\left\{D_{+}, \bar{D}_{+}\right\}=i \partial \quad\left\{D_{-}, \bar{D}_{-}\right\}=-i \bar{\partial}
$$

Chiral and antichiral superfields $\Psi$ and $\bar{\Psi}=\Psi^{*}$ are subject to the constraints

$$
\bar{D}_{ \pm} \Psi=0 \quad D_{ \pm} \bar{\Psi}=0
$$

Then one defines new coordinates

$$
\begin{aligned}
\theta_{1} & =\frac{1}{2}\left(\theta_{+}+\bar{\theta}_{+}\right) & \bar{\theta}_{1} & =\frac{1}{2}\left(\theta_{-}+\bar{\theta}_{-}\right) \\
\theta_{2} & =\frac{i}{2}\left(\theta_{+}-\bar{\theta}_{+}\right) & \bar{\theta}_{2} & =\frac{i}{2}\left(\theta_{-}-\bar{\theta}_{-}\right)
\end{aligned}
$$

so that given a $N=1$ superfield $\Phi\left(\theta_{1}, \bar{\theta}_{1}\right)$, the chirality condition in eq.(8) allows to reconstruct a $N=2$ superfield

$$
\Psi\left(\theta_{+}, \theta_{-}, \bar{\theta}_{+}, \bar{\theta}_{-}\right)=e^{-2 \theta_{1} \theta_{2} \partial+2 \bar{\theta}_{1} \bar{\theta}_{2} \bar{\partial}} \Phi\left(\theta_{1}-i \theta_{2}, \bar{\theta}_{1}-i \bar{\theta}_{2}\right)
$$

Applying this procedure to the $N=1$ superfields $\Phi_{i}^{( \pm)}$, one defines the following $N=2$ chiral and antichiral superfields

$$
\begin{array}{ll}
\Phi_{i}^{(+)} \rightarrow \Psi_{i}^{(+)} & \Phi_{i}^{(+) *} \rightarrow \bar{\Psi}_{i}^{(+)} \\
\Phi_{i}^{(-) *} \rightarrow \Psi_{i}^{(-)} & \Phi_{i}^{(-)} \rightarrow \bar{\Psi}_{i}^{(-)}
\end{array}
$$

Thus the action in eq.(四) can be reexpressed in $N=2$ superspace

$$
S=\frac{1}{\beta^{2}} \int d^{2} z d^{4} \theta \quad K_{i j}\left[\Psi_{i}^{(+)} \bar{\Psi}_{j}^{(-)}+\Psi_{i}^{(-)} \bar{\Psi}_{j}^{(+)}\right]+\frac{1}{\beta^{2}} \int d^{2} z d^{2} \theta \quad W(\Psi)+\frac{1}{\beta^{2}} \int d^{2} z d^{2} \bar{\theta} W(\bar{\Psi})
$$

where the superpotential $W$ is given by

$$
W(\Psi)=V\left(\Psi^{(+)}\right)+V\left(\Psi^{(-)}\right)
$$

We address now the issue of twisting the theory into a topological sector. To this end it is convenient to rewrite the action in components and eliminate the auxiliary fields through the field equations $F_{i}^{( \pm)}=-K_{i j}^{-1} \frac{\partial V}{\partial \phi_{j}^{(\mp)}}$

$$
\begin{aligned}
S=\frac{1}{\beta^{2}} \int d^{2} z & \left\{K_{i j}\left[\partial \phi_{i}^{(-)} \bar{\partial} \phi_{j}^{(+)}+\frac{i}{2} \psi_{i}^{(-)} \bar{\partial} \psi_{j}^{(+)}-\frac{i}{2} \bar{\psi}_{i}^{(-)} \partial \bar{\psi}_{j}^{(+)}\right]-\frac{\partial V}{\partial \phi_{i}^{(+)}} K_{i j}^{-1} \frac{\partial V}{\partial \phi_{j}^{(-)}}\right. \\
& \left.+\frac{1}{2} \bar{\psi}_{i}^{(+)} \psi_{j}^{(+)} \frac{\partial^{2} V}{\partial \phi_{i}^{(+)} \partial \phi_{j}^{(+)}}+\frac{1}{2} \bar{\psi}_{i}^{(-)} \psi_{j}^{(-)} \frac{\partial^{2} V}{\partial \phi_{i}^{(-)} \partial \phi_{j}^{(-)}}+\text {h.c. }\right\}
\end{aligned}
$$


Then it is clear that these models possess a $\Lambda \equiv O(1,1)$ symmetry

$$
\begin{aligned}
\delta_{\Lambda} \phi_{i}^{( \pm)} & =0 \\
\delta_{\Lambda} \psi_{i}^{( \pm)} & = \pm \omega \psi_{i}^{( \pm)} \\
\delta_{\Lambda} \bar{\psi}_{i}^{( \pm)} & =\mp \omega \bar{\psi}_{i}^{( \pm)}
\end{aligned}
$$

where $\omega$ is a real parameter. Since this internal symmetry group is isomorphic to the Lorentz group $L$

$$
\begin{aligned}
\delta_{L} \phi_{i}^{( \pm)} & =0 \\
\delta_{L} \psi_{i}^{( \pm)} & =\frac{\lambda}{2} \psi_{i}^{( \pm)} \\
\delta_{L} \bar{\psi}_{i}^{( \pm)} & =-\frac{\lambda}{2} \bar{\psi}_{i}^{( \pm)}
\end{aligned}
$$

new transformations can be defined corresponding to a twisted Lorentz group $L^{\prime} \sim(\Lambda \otimes$ $L)_{\text {diag. }}$. With the choice $\omega=-\frac{\lambda}{2}$ one obtains

$$
\begin{aligned}
\delta_{L^{\prime}} \phi_{i}^{( \pm)} & =0 \\
\delta_{L^{\prime}} \psi_{i}^{(-)} & =\lambda \psi_{i}^{(-)} \\
\delta_{L^{\prime}} \psi_{i}^{(+)} & =0 \\
\delta_{L^{\prime}} \bar{\psi}_{i}^{(-)} & =-\lambda \bar{\psi}_{i}^{(-)} \\
\delta_{L^{\prime}} \bar{\psi}_{i}^{(+)} & =0
\end{aligned}
$$

Therefore, with respect to the Lorentz group $L^{\prime}$ the new spin assignment on the spinors is

$$
\begin{array}{llll}
\psi_{i}^{(+)}: & s^{\prime}=0 & \bar{\psi}_{i}^{(+)}: & s^{\prime}=0 \\
\psi_{i}^{(-)}: & : s^{\prime}=1 & \bar{\psi}_{i}^{(-)}: & s^{\prime}=-1
\end{array}
$$

(The twist corresponding to the choice $\omega=\frac{\lambda}{2}$ would simply interchange the spin assignment between $\psi^{(+)}, \bar{\psi}^{(+)}$and $\psi^{(-)}, \bar{\psi}^{(-)}$.) It is now possible to construct a nihilpotent linear combination of the supersymmetry charges with $s^{\prime}=0$. Indeed let us consider the charge

$$
Q=\frac{1}{\sqrt{2}}\left[Q_{L}^{+}-i Q_{R}^{+}+i\left(Q_{L}^{-}-i Q_{R}^{-}\right) J\right]
$$

Using eq.(6) we obtain the following transformations on the component fields

$$
\begin{aligned}
\delta \phi_{i}^{(+)} & =\eta\left[i \psi_{i}^{(+)}+\bar{\psi}_{i}^{(+)}\right] \\
\delta \phi_{i}^{(-)} & =0 \\
\delta \psi_{i}^{(+)} & =-2 \eta K_{i j}^{-1} \frac{\partial V}{\partial \phi_{j}^{(-)}}
\end{aligned}
$$




$$
\begin{aligned}
\delta \psi_{i}^{(-)} & =-2 \eta \partial \phi_{i}^{(-)} \\
\delta \bar{\psi}_{i}^{(+)} & =2 i \eta K_{i j}^{-1} \frac{\partial V}{\partial \phi_{j}^{(-)}} \\
\delta \bar{\psi}_{i}^{(-)} & =-2 i \eta \bar{\partial} \phi_{i}^{(-)}
\end{aligned}
$$

With respect to the twisted Lorentz group the parameter $\eta$ has spin zero. Moreover $Q^{2}=0, Q^{\dagger}=Q$ so that the charge in eq.(19) represents the BRST operator of the twisted theory. It can be rewritten as

$$
Q=\frac{1}{\beta^{2}} \int d x\left[\frac{i}{\sqrt{2}} K_{i j}\left(i \psi_{i}^{(+)} \partial \phi_{j}^{(-)}+\bar{\psi}_{i}^{(+)} \bar{\partial} \phi_{j}^{(-)}\right)-\frac{1}{\sqrt{2}}\left(\psi_{i}^{(-)}+i \bar{\psi}_{i}^{(-)}\right) \frac{\partial V}{\partial \phi_{i}^{(-)}}+\text {h.c. }\right]
$$

The holomorphic component of the stress-energy tensor

$$
T=-K_{i j} \partial \phi_{i}^{(-)} \partial \phi_{j}^{(+)}-\frac{i}{2} K_{i j} \psi_{i}^{(-)} \partial \psi_{j}^{(+)}+\text {h.c. }
$$

satisfies the conservation equation

$$
\bar{\partial} T=\partial \Theta
$$

where the trace is given by

$$
\Theta=\frac{\partial V}{\partial \phi_{i}^{(+)}} K_{i j}^{-1} \frac{\partial V}{\partial \phi_{j}^{(-)}}+\frac{1}{2} \psi_{i}^{(+)} \bar{\psi}_{j}^{(+)} \frac{\partial^{2} V}{\partial \phi_{i}^{(+)} \partial \phi_{j}^{(+)}}+\text {h.c. }
$$

It is easy to check that the corresponding central charge is zero and $T, \Theta$ can be written as BRST anticommutators

$$
\begin{aligned}
T & =\left\{Q, \frac{1}{2} K_{i j} \psi_{i}^{(-)} \partial \phi_{j}^{(+)}+\text {h.c. }\right\} \\
\Theta & =\left\{Q,-\frac{1}{2} \psi_{i}^{(+)} \frac{\partial V}{\partial \phi_{i}^{(+)}}+\text {h.c. }\right\}
\end{aligned}
$$

The BRST structure of our twisted theory shares some similarities with the one considered in Ref. 110. In both cases the topological invariance is a consequence of eq.(25), while the lagrangian is not $Q$-exact.

We notice that the twist can be performed even if we impose reality conditions $\Phi_{i}^{( \pm) *}=$ $\Phi_{i}^{( \pm)}$on the superfields in eq.(化). In fact with this choice the theory has not a genuine $N=2$ supersymmetry with a $U(1)$ generator, but it is still non-unitary and it has an extended superalgebra containing a $O(1,1)$. On the other hand the conditions $\Phi_{i}^{(+) *}=$ $\Phi_{i}^{(-)}$and $K_{i j}$ positive definite restore unitarity but the $O(1,1)$ invariance is lost.

Now we study the physical content of these topological theories. The physical states are given by cohomology classes of the BRST operator. The BRST transformations in eq.(20) imply that $\frac{\partial V}{\partial \phi_{i}^{(-)}}$is $Q$-exact so that its expectation value on physical states 
vanishes. This condition is satisfied at the critical points of the potential $V\left(\phi^{(-)}\right)$and it ensures the nihilpotency of the BRST operator at the quantum level. As a consequence of the fact that the stress-energy tensor is $Q$-exact it follows that the correlation functions of the observables are independent of the space-time coordinates. Moreover since the theory contains an equal number of bosons and ghost-like fields all particle excitations decouple. This guarantees the absence of negative norm states which appear at the lagrangian level due to the fact that the kinetic term is not positive definite. The physical spectrum is characterized by observables associated to BRST invariant operators. These are the topological charge $\mathcal{T}=\int_{M} d x \frac{\partial \phi}{\partial x}^{(+)}$, where $M$ is the space line, and the operator $\mathcal{O}\left(P^{(+)}\right)$which maps one critical point into another, being $P^{(+)}$the canonical momentum conjugated to $\phi^{(-)}$. States in different topological sectors are orthogonal.

The physical spectrum can be explicitly determined in a weak-coupling expansion around a critical point. Deforming the background solution into a nearby one $\phi_{i}^{( \pm)}+\delta \phi_{i}^{( \pm)}$ we obtain the linearized bosonic field equations

$$
K_{i j} \partial \bar{\partial} \delta \phi_{j}^{( \pm)}+\frac{\partial^{2} V}{\partial \phi_{i}^{(\mp)} \partial \phi_{j}^{(\mp)}} K_{j l}^{-1} \frac{\partial^{2} V}{\partial \phi_{l}^{( \pm)} \partial \phi_{m}^{( \pm)}} \delta \phi_{m}^{( \pm)}=0
$$

These equations are satisfied also by the $\psi_{i}^{(+)}, \bar{\psi}_{i}^{(+)}$fermions which are then deformations of the bosonic fields. Since the combination $i \psi_{i}^{(+)}+\bar{\psi}_{i}^{(+)}$is the BRST transform of $\phi_{i}^{(+)}$ one can use the BRST invariance of the theory to deform continuously $\phi^{(+)}$-backgrounds with the same topological charge. Therefore one can perform a weak-coupling expansion around one representative within each sector.

In order to compute the relevant physical quantities one has to select a specific form of the potential. The simplest case is the free theory described by the lagrangian in eq.(14) where we set $V=0, \beta=1$ and compactify the fields on $M=S^{1}$ with periodic boundary conditions. In this case the topological charge is trivial and we will show that the only physical quantity is the momentum $p^{(+)}=\frac{1}{\pi} \int_{S^{1}} d x P^{(+)}$, where $P^{(+)}=\frac{1}{2} \frac{\partial \phi}{\partial t}^{(+)}$. It is sufficient to analyze the case of two real bosonic fields $\phi^{( \pm)}$and their fermionic partners with metric $K=1$. The fields admit a mode expansion

$$
\begin{aligned}
& \phi_{L}^{( \pm)}=q_{L}^{( \pm)}+p_{L}^{( \pm)}(t+x)+\sum_{n<0} \frac{1}{\sqrt{2 \pi}|n|}\left[a_{n}^{( \pm)} e^{i n(t+x)}+a_{n}^{( \pm)^{\dagger}} e^{-i n(t+x)}\right] \\
& \phi_{R}^{( \pm)}=q_{R}^{( \pm)}+p_{R}^{( \pm)}(t-x)+\sum_{n>0} \frac{1}{\sqrt{2 \pi}|n|}\left[a_{n}^{( \pm)} e^{i n(x-t)}+a_{n}^{( \pm)^{\dagger}} e^{-i n(x-t)}\right] \\
& \psi^{( \pm)}=\frac{2^{\frac{1}{4}}}{\sqrt{\pi}} b_{0}^{( \pm)}+\frac{2^{\frac{1}{4}}}{\sqrt{\pi}} \sum_{n<0}\left[b_{n}^{( \pm)} e^{i n(t+x)}+b_{n}^{( \pm)^{\dagger}} e^{-i n(t+x)}\right] \\
& \bar{\psi}^{( \pm)}=i \frac{2^{\frac{1}{4}}}{\sqrt{\pi}} \bar{b}_{0}^{( \pm)}+i \frac{2^{\frac{1}{4}}}{\sqrt{\pi}} \sum_{n>0}\left[\bar{b}_{n}^{( \pm)} e^{i n(x-t)}+\left(\bar{b}_{n}^{( \pm)}\right)^{\dagger} e^{-i n(x-t)}\right]
\end{aligned}
$$


where $q_{L}^{( \pm)}=q_{R}^{( \pm)} \equiv \frac{q^{( \pm)}}{2}, p_{L}^{( \pm)}=p_{R}^{( \pm)} \equiv \frac{p^{( \pm)}}{2}$. The canonical commutation relations are

$$
\begin{aligned}
{\left[q^{( \pm)}, p^{(\mp)}\right] } & =\frac{i}{\pi} & & {\left[a_{m}^{( \pm)}, a_{n}^{(\mp)^{\dagger}}\right]=|n| \delta_{m, n} \quad m, n \in Z-\{0\} } \\
\left\{b_{m}^{( \pm)}, b_{n}^{(\mp)}\right\} & =\delta_{m, n} & & m, n \leq 0 \\
\left\{\bar{b}_{m}^{( \pm)},\left(\bar{b}_{n}^{(\mp)}\right)^{\dagger}\right\} & =\delta_{m, n} & & m, n \geq 0
\end{aligned}
$$

and $b_{0}^{( \pm)^{\dagger}}=b_{0}^{( \pm)},\left(\bar{b}_{0}^{( \pm)}\right)^{\dagger}=\bar{b}_{0}^{( \pm)}$. Setting the potential to zero in eq.(21) we obtain the BRST charge

$$
\begin{aligned}
Q= & \frac{1}{\sqrt{2}} \int_{0}^{2 \pi} d x\left[-\psi^{(+)} \partial \phi^{(-)}+i \bar{\psi}^{(+)} \bar{\partial} \phi^{(-)}\right] \\
= & -\frac{2^{\frac{1}{4}}}{\sqrt{\pi}}\left[2 \pi b_{0}^{(+)} p_{L}^{(-)}+2 \pi \bar{b}_{0}^{(+)} p_{R}^{(-)}+i \sqrt{2 \pi} \sum_{n<0}\left(b_{n}^{(+)} a_{n}^{\left.(-)^{\dagger}-b_{n}^{(+)}{ }^{\dagger} a_{n}^{(-)}\right)}\right.\right. \\
& \left.+i \sqrt{2 \pi} \sum_{n>0}\left(\bar{b}_{n}^{(+)} a_{n}^{(-)}{ }^{\dagger}-\left(\bar{b}_{n}^{(+)}\right)^{\dagger} a_{n}^{(-)}\right)\right]
\end{aligned}
$$

The Fock vacuum is defined to be annihilated by $a_{n}^{( \pm)}, b_{n}^{( \pm)}$and $\bar{b}_{n}^{( \pm)}, n \neq 0$ oscillators, and it carries momentum $p^{( \pm)}$.

Let us show first that all particle excitations are cohomologically trivial. We start by considering the left-moving sector. The oscillators satisfy the following BRST transformations

$$
\left[Q_{L}, a_{n}^{(+)}\right]=-i n b_{n}^{(+)} \quad\left\{Q_{L}, b_{n}^{(-)}\right\}=i a_{n}^{(-)} \quad n<0
$$

where we have defined

$$
Q_{L}=i \sum_{n<0}\left(b_{n}^{(+)^{\dagger}} a_{n}^{(-)}-b_{n}^{(+)} a_{n}^{(-)^{\dagger}}\right)
$$

Denoting by $P_{0}$ the projector on zero-particle states, the projector on $N$-particle states is

$$
P_{N}=\frac{1}{N} \sum_{n<0}\left[\frac{1}{|n|} a_{n}^{(+)^{\dagger}} P_{N-1} a_{n}^{(-)}+\frac{1}{|n|} a_{n}^{(-)^{\dagger}} P_{N-1} a_{n}^{(+)}+b_{n}^{(+)}{ }^{\dagger} P_{N-1} b_{n}^{(-)}+b_{n}^{(-)^{\dagger}} P_{N-1} b_{n}^{(+)}\right]
$$

It is easy to show that $P_{N}=\left\{Q_{L}, R_{N}\right\}$, where

$$
R_{N}=\frac{i}{N} \sum_{n<0} \frac{1}{|n|}\left[\left(b_{n}^{(-)}\right)^{\dagger} P_{N-1} a_{n}^{(+)}-\left(a_{n}^{(+)}\right)^{\dagger} P_{N-1} b_{n}^{(-)}\right]
$$

From the condition $Q_{L} \mid$ phys $\rangle=0$ it follows that $\mid$ phys $\rangle$ is cohomologically equivalent to $P_{0} \mid$ phys $\rangle$. Extending this analysis to the right-moving sector one shows that all particle excitations are unphysical.

Now we focus on the zero modes. We define

$$
Q_{0}=p^{(-)}\left[b_{0}^{(+)}+\bar{b}_{0}^{(+)}\right]
$$


and expand any state on the $b_{0}$-modes

$$
|\Omega\rangle=\left|\Omega_{1}\right\rangle+b_{0}^{(+)}\left|\Omega_{2}\right\rangle+\bar{b}_{0}^{(+)}\left|\Omega_{3}\right\rangle+b_{0}^{(+)} \bar{b}_{0}^{(+)}\left|\Omega_{4}\right\rangle
$$

The physical state condition implies

$$
p^{(-)}\left|\Omega_{1}\right\rangle=0 \quad p^{(-)}\left|\Omega_{2}\right\rangle=p^{(-)}\left|\Omega_{3}\right\rangle
$$

so that if $p^{(-)} \neq 0$ the states in eq.(35) are cohomologically trivial (for instance the quantity $b_{0}^{(+)} \bar{b}_{0}^{(+)}\left|\Omega_{4}\right\rangle$ can be written as $\left.-Q_{0} \frac{1}{p^{(-)}} b_{0}^{(+)}\left|\Omega_{4}\right\rangle\right)$. Therefore the physical states are characterized by the conditions $p^{(-)}=0$ and $p^{(+)}$arbitrary. Since the potential is trivial, the operator which maps the set of critical points into itself is the translation operator $p^{(+)}$. The zero-modes $q^{( \pm)}$can be interpreted as the coordinates of a two-dimensional target space with metric $\eta_{+-}=1$. We stress that this theory is not topological in target space since deformations of $\eta_{\mu \nu}$ in the action are not $Q$-exact. Therefore propagation on target space is possible and in fact the physical states $\left|p^{(+)}\right\rangle$describe a massless bosonic field right-moving on this space. In this respect they resemble $D=2$ string theories where a massless mode propagates on a two-dimensional space-time (cfr. the critical $N=2$ string [11] or the bosonic $c=1$ string [12]).

The analysis can be extended to the toroidal compactification of the free model. In this case the bosonic fields take values on a circle

$$
\phi^{( \pm)} \equiv \phi^{( \pm)}+2 \pi R n^{( \pm)}
$$

where the winding numbers $n^{( \pm)}$are related to the topological charges $\mathcal{T}^{( \pm)}=2 \pi R n^{( \pm)}$. The mode expansion of the fields is given in eq.(27) where now the left and right components of the zero modes are independent and $p_{L}-p_{R}$ is the winding number operator.

The decoupling of the particle excitations can be proven as in the previous example. In the zero-mode sector we define

$$
Q_{0}=b_{0}^{(+)} p_{L}^{(-)}+\bar{b}_{0}^{(+)} p_{R}^{(-)}
$$

Thus the conditions in eq. (36) become

$$
p_{L, R}^{(-)}\left|\Omega_{1}\right\rangle=0 \quad p_{R}^{(-)}\left|\Omega_{2}\right\rangle=p_{L}^{(-)}\left|\Omega_{3}\right\rangle
$$

Again one can easily show that the states are cohomologically trivial whenever $p_{L}^{(-)} \neq 0$ or $p_{R}^{(-)} \neq 0$. Therefore the physical states are characterized by $p_{L, R}^{(-)}=0$ and $p_{L, R}^{(+)}=$ $\frac{m}{2 \pi R} \pm \frac{n^{(+)}}{2} R$. They describe solitons with winding number $n^{(+)}$and momentum $\frac{m}{\pi R}$.

Now we turn to the interacting theories and look for a potential which admits an infinite number of critical points with solitonic configurations interpolating between them. 
What we need is a $N=2$ complexified supersymmetric sine-Gordon theory or a generalization of it. In fact a whole class of models is available: they are the supersymmetric Toda theories based on the affine superalgebra $A^{(1)}(n, n)$ [13, 8, 14]. This superalgebra has rank $r=2 n$ and it admits a purely fermionic set of $2 n+2$ roots, which can be represented in terms of $2 n$-dimensional complex vectors. The roots $\vec{\alpha}_{j}$ with $j=1, \ldots, 2 n$ realize the Cartan matrix of the $A(n, n-1)$ superalgebra, i.e.

$$
\vec{\alpha}_{j}^{2}=0 \quad \vec{\alpha}_{i} \cdot \vec{\alpha}_{j}=2(-1)^{i+1} \delta_{j, i+1}+2(-1)^{i} \delta_{j, i-1}
$$

and $\vec{\alpha}_{2 n+1}=-\sum_{j=1}^{n} \vec{\alpha}_{2 j-1}, \vec{\alpha}_{0}=-\sum_{j=1}^{n} \vec{\alpha}_{2 j}$. The Toda equations of motion for the $2 n$ complex $N=1$ superfields $\Phi^{a}$

$$
2 \bar{D} D \Phi^{a}+\sum_{i=0}^{2 n+1} \alpha_{i}^{a} e^{\vec{\alpha}_{i} \cdot \vec{\Phi}}=0
$$

can be derived from a real, non-unitary lagrangian 15

$$
S=\frac{1}{\beta^{2}} \int d^{2} z d^{2} \theta\left[D \vec{\Phi} \cdot \bar{D} \vec{\Phi}+\sum_{i=0}^{2 n+1} e^{\vec{\alpha}_{i} \cdot \vec{\Phi}}+\text { h.c. }\right]
$$

We now perform the field redefinitions introduced in Ref. [8]

$$
\begin{array}{lll}
\Phi_{i}^{(+)}=\vec{\alpha}_{i} \cdot \vec{\Phi} & \Phi_{i}^{(-)}=\vec{\alpha}_{\sigma(i)} \cdot \vec{\Phi} & \\
\Phi_{i}^{(-)}=\vec{\alpha}_{i} \cdot \vec{\Phi} & \Phi_{i}^{(+)}=\vec{\alpha}_{\sigma(i)} \cdot \vec{\Phi} &
\end{array}
$$

where $\sigma(i) \equiv 2 n+1-i, i=1, \ldots, n$, generates an automorphism $\alpha_{i} \rightarrow \alpha_{\sigma(i)}$ of the Dynkin diagram of the $A(n, n-1)$ superalgebra. In terms of the new complex superfields the action in eq. (42) takes the general form in eq.(14) where

$$
V\left(\Phi^{( \pm)}\right)=\sum_{i=1}^{n} e^{\Phi_{i}^{( \pm)}}+e^{-\sum_{i=1}^{n} \Phi_{i}^{( \pm)}}
$$

and $K_{i j}$ is the $n \times n$ matrix

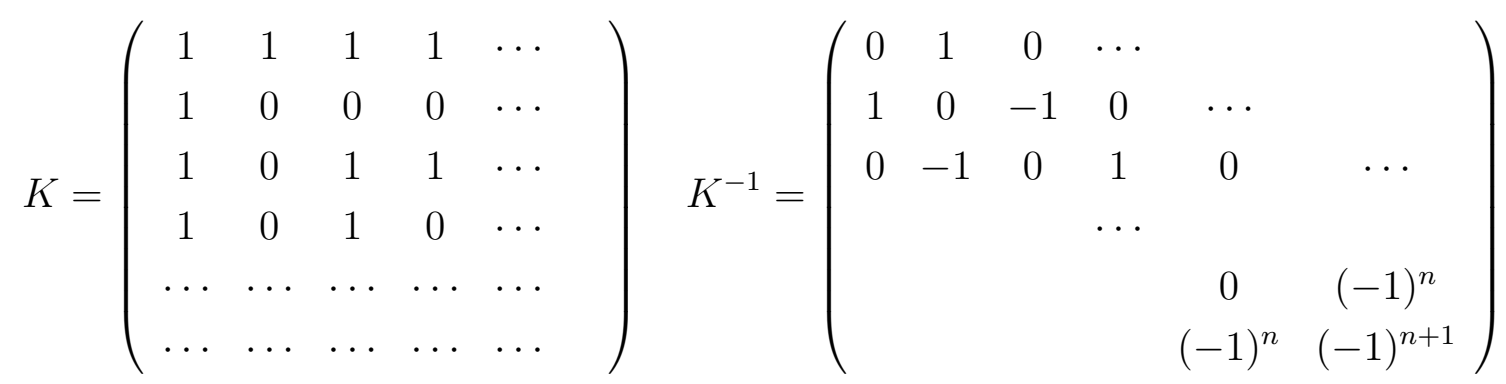

First we discuss the existence of solitonic configurations which can be determined setting the fermion fields to zero and searching for nontrivial, finite energy solutions of 
the bosonic equations of motion. As observed in Ref. [8] it is convenient to redefine the bosonic variables

$$
\begin{aligned}
\phi_{2 j-1}^{(-)}=i\left(\xi_{j}^{(1)}-\xi_{j}^{(2)}\right) & \phi_{2 j-1}^{(+)}=i\left(\xi_{n+1-j}^{(2)}-\xi_{n+2-j}^{(1)}\right) & j=1, \ldots,\left[\frac{n+1}{2}\right] \\
\phi_{2 j}^{(-)}=i\left(\xi_{n+1-j}^{(1)}-\xi_{n+1-j}^{(2)}\right) & \phi_{2 j}^{(+)}=i\left(\xi_{j}^{(2)}-\xi_{j+1}^{(1)}\right) & j=1, \ldots,\left[\frac{n}{2}\right]
\end{aligned}
$$

with $\xi_{0}^{(1)}=\xi_{n+1}^{(1)}=-\sum_{j=1}^{n} \xi_{j}^{(1)}, \xi_{0}^{(2)}=\xi_{n+1}^{(2)}=-\sum_{j=1}^{n} \xi_{j}^{(2)}$. It is easy to check that the bosonic lagrangian reexpressed in terms of the $\xi$-fields becomes

$$
\mathcal{L}_{\mathrm{B}}=\mathcal{L}\left(\xi^{(2)}\right)-\mathcal{L}\left(\xi^{(1)}\right)+\text { h.c. }
$$

where $\mathcal{L}$ is the Toda lagrangian for the bosonic $a_{n}^{(1)}$ theory with imaginary coupling

$$
\mathcal{L}=\frac{1}{\beta^{2}} \sum_{j=0}^{n}\left[\frac{1}{2} \partial \xi_{j} \bar{\partial} \xi_{j}+e^{i\left(\xi_{j}-\xi_{j+1}\right)}\right]
$$

We can then borrow all the results that have been obtained on solitonic solutions for the $a_{n}^{(1)}$ complex Toda theory [6, 7]. It has been shown that even though the fields are complex the solitons have real energy and momentum. Since they describe finite energy configurations they satisfy the boundary conditions $\vec{\xi}(x= \pm \infty) \in 2 \pi \Lambda_{\omega}$ where $\Lambda_{\omega}$ is the weight lattice of the algebra. In particular the one-soliton static solutions are

$$
\xi_{j}^{(a)}=i \log \frac{\left(1+e^{\sigma_{a} x-\lambda} \omega_{a}^{j}\right)}{\left(1+e^{\sigma_{a} x-\lambda} \omega_{a}^{j-1}\right)} \quad a=1, \ldots, n
$$

where $\omega_{a} \equiv e^{\frac{2 \pi a}{n+1} i}, \sigma_{a}=2 \sqrt{2} \sin \frac{\pi a}{n+1}$ and $\lambda$ is a complex parameter. The solitons of the supersymmetric $A^{(1)}(n, n)$ models are given by the configurations $\left(\xi^{(1)}, \xi^{(2)}\right)$, with $\xi^{(1)}$ and $\xi^{(2)}$ solitonic solutions of the corresponding bosonic theory.

Now we perform the twist and concentrate on the BRST invariant theory. From the general discussion we know that $\phi_{i}^{(-)}$must be an extremum of the potential $V\left(\phi^{(-)}\right)$. In terms of the $\xi$ fields introduced in eq. (46) this gives

$$
\vec{\xi}^{(1)}=\vec{\xi}^{(2)} \quad \bmod 2 \pi \Lambda_{\omega}
$$

Therefore the topological $A^{(1)}(n, n)$ theory has the same solitonic spectrum of the $a_{n}^{(1)}$ theory. The energy-momentum tensor of these configurations vanishes in agreement with topological invariance.

In the weak coupling expansion around a solitonic background the bosonic field deformations and the fermionic fields $\psi_{i}^{(+)}, \bar{\psi}_{i}^{(+)}$satisfy the linearized $a_{n}^{(1)}$ Toda field equations. The normal modes of the solutions can be determined explicitly [16, 17] since 
multi-solitonic configurations are known [6, 7]. Using the canonical BRST analysis 18 it is easy to show that the particle excitations are cohomologically trivial, whereas the study of the zero-mode sector requires a careful treatment with collective coordinates. We intend to report on this in a future publication. 


\section{References}

[1] V. Knizhnik, A. Polyakov and A.B. Zamolodchikov, Mod. Phys. Lett. A3 (1988) 819.

[2] E. Brézin and V. Kazakov, Phys. Lett. 236B (1990) 144;

M. Douglas and S. Shenker, Nucl. Phys. B335 (1990) 635;

D.J. Gross and A.A. Migdal, Phys. Rev. Lett. 64 (1990) 127.

[3] E. Witten, Nucl. Phys. B340 (1990) 281.

[4] K. Li, Nucl. Phys. B354 (1991) 711; Nucl. Phys. B354 (1991) 725;

R. Dijkgraaf, E. Verlinde and H. Verlinde, Nucl. Phys. B352 (1991) 59.

[5] S. Mukhi and C. Vafa, "Two-dimensional black hole as a topological coset model of $c=1$ string theory", HUTP-93/A002, TIFR/TH/93-01;

O. Aharony, O. Ganor, J. Sonnenschein and S. Yankielowicz, " $c=1$ string theory as a topological $\frac{G}{G}$ model", TAUP-2032-93 (February 1993).

[6] T. Hollowood, Nucl. Phys. B384 (1992) 523.

[7] H.C. Liao, D. Olive and N. Turok, "Topological solitons in $A_{r}$ Affine Toda Theory", Imperial/TP/91-92/34 (September 1992).

[8] J. Evans and T. Hollowood, Phys. Lett. 293B (1992) 100.

[9] S.J. Gates Jr., C.M. Hull and M. Roček, Nucl. Phys. B248 (1984) 157;

M.T. Grisaru and D. Zanon, Phys. Lett. 184B (1987) 209.

[10] C. Vafa, Mod. Phys. Lett. A6 (1991) 337.

[11] M. Ademollo, L. Brink, A. D’Adda, R. D'Auria, E. Napolitano, S. Sciuto, E. Del Giudice, P. Di Vecchia, S. Ferrara, F. Gliozzi, R. Musto, R. Pettorino and J.H. Schwarz, Nucl. Phys. B111 (1976) 77;

H. Ooguri and C. Vafa, Mod. Phys. Lett. A5 (1990) 1389.

[12] J.Polchinski, Nucl. Phys. B346 (1990) 253;

A.M. Polyakov, Mod. Phys. Lett. A6 (1991) 635;

E. Witten and B. Zwiebach, Nucl. Phys. B377 (1992) 55.

[13] A. Gualzetti, S. Penati and D. Zanon, "Quantum conserved currents in supersymmetric Toda theories", IFUM 426/FT (June 1992) hep-th/9207018, to appear in Nucl. Phys. B. 
[14] S. Penati and D. Zanon, "Quantum symmetries in supersymmetric Toda theories", IFUM 435/FT (November 1992) hep-th/9211033, to appear in the Proceedings of "String theory, quantum gravity and the unification of the fundamental interactions", Roma - September 1992.

[15] J. Evans, "Complex Toda theories and twisted reality conditions", OUTP-91-39P.

[16] R.F. Dashen, B. Hasslacher and A. Neveu, Phys. Rev. D11 (1975) 3424.

[17] T. Hollowood, Phys. Lett. 300B (1993) 73.

[18] T. Kugo and I. Ojima, Phys. Lett. 73B (1978) 459; Suppl. Prog. Theor. Phys. 66 (1979) 1. 